\title{
Electrically tunable spatially variable switching in ferroelectric liquid crystal/water system
}

\author{
A. Choudhary, ${ }^{1,2}$ I. Coondoo, ${ }^{1}$ J. Prakash, ${ }^{1, \text { a) }}$ K. Sreenivas,${ }^{2}$ and A. M. Biradar ${ }^{1, b)}$ \\ ${ }^{1}$ National Physical Laboratory, Dr. K. S. Krishnan Road, New Delhi 110012, India \\ ${ }^{2}$ Department of Physics and Astrophysics, University of Delhi, Delhi 110007, India
}

(Received 31 December 2008; accepted 8 April 2009; published online 29 April 2009)

\begin{abstract}
An unusual switching phenomenon in the region outside conducting patterned area in ferroelectric liquid crystal (FLC) containing about 1-2 wt \% of water has been observed. The presence of water in the studied heterogeneous system was confirmed by Fourier transform infrared spectroscopy. The observed optical studies have been emphasized on the "spatially variable switching" phenomenon of the molecules in the nonconducting region of the cell. The observed phenomenon is due to diffusion of water between the smectic layers of the FLC and the interaction of the curved electric field lines with the FLC molecules in the nonconducting region. () 2009 American Institute of Physics. [DOI: $10.1063 / 1.3126960$ ]
\end{abstract}

Conjugation of water with amphiphilic and other organic compounds results in interesting optical and electrical properties of the system-the liquid crystal (LC) phase. ${ }^{1-4}$ Lyotropic LCs, which are formed by addition of water to various amphiphilic materials, seem to play an important role in many biological systems including cell membranes. ${ }^{5-7}$ The $\mathrm{x}$-ray diffraction studies on the two most common phasesneat and middle - of lyotropic LCs have revealed that the neat phase is lamellar and the structure consists of alternate layers of the lyotropic mesogens and water. ${ }^{1,5,8}$

Heterogeneous systems consisting of a LC and an immiscible liquid are of interest from both scientific perspective and for potential applications. The properties exhibited by such dispersions of chemically dissimilar phasesisotropic liquid and anisotropic LC host reflect an interesting microscopic structural organization due to the interactions between the constituents. ${ }^{9,10}$ Several investigations have been carried out including studies on isotropic droplets in nematics, ${ }^{11,12}$ nematic emulsions, ${ }^{13,14}$ nematic LC/water interfaces, ${ }^{15,16}$ etc. in such heterogeneous systems. The thermotropic LC hosts considered so far have been nematics, although little work has been reported on other mesophases like the smectics as well. ${ }^{17-19}$ The common and most striking feature of the smectic phase is the existence of a layered structure. ${ }^{10}$ The molecular centers are on average arranged in equidistant planes thus forming a stratified conformation rendering it both the long-range translational order plus local orientational order. The colloidal interaction between the interfaces of the constituents of a heterogeneous system plays a characteristic role in determining their unique properties. The interfacial surface tension $(\gamma)$ at the interface between LC/air ${ }^{1,20,21}$ and positive slope $(d \gamma / d T), T$ is the temperature, indicating the excess order at the interface LC/liquid ${ }^{15,16,22-24}$ are well reported.

Based on these reports, it would be interesting to study the effect on various electro-optical properties of a smectic LC due to the diffusion of an isotropic liquid such as water,

\footnotetext{
a) Also at Instrument Design Development Center, I I T Delhi, New Delhi 110016, India.

b) Author to whom correspondence should be addressed. Electronic mail: abiradar@mail.nplindia.ernet.in.
}

ethanol, etc. in between the smectic layers. With this objective, we in this letter, investigate the effect of mixing of an immiscible isotropic liquid (i.e., water) into the ferroelectric LC (FLC) namely CS1026. We anticipate that the weakening of the electrostatic interactions due to the water layer between the smectic layers of the FLC and the interfacial surface tension induces the interesting "spatially variable switching" phenomenon.

In cell fabrication, circular rings (diameter $2.32 \mathrm{~mm}$ ) of low resistant (10-18 $\Omega / \square)$ indium tin oxide film coated on optically flat glass substrate were used as electrodes. The substrates were treated with adhesion promoter (phenyl trichlorosilane in toluene) followed by nylon $6 / 6$ and rubbed (by LCBM, Irwin, USA) unidirectionally to get homogeneous alignment of LC molecules. Photoresist was used as a spacer $(\sim 6 \mu \mathrm{m})$ between substrates by negative photolithography. The phase sequence of the FLC material (CS1026, Chisso Corp., Tokyo, Japan) is as follows:

$$
\begin{gathered}
-7^{\circ} \mathrm{C} \quad 64^{\circ} \mathrm{C} \quad 82^{\circ} \mathrm{C} \quad 91^{\circ} \mathrm{C} \\
\text { cryst } \leftrightarrow \mathrm{SmC}^{*} \leftrightarrow \mathrm{SmA} \leftrightarrow \mathrm{N} \leftrightarrow \text { iso. }
\end{gathered}
$$

Since water does not dissolve into the studied FLC material at room temperature, one has to diffuse it into LC at higher temperature in nematic phase. ${ }^{19,25}$ The system of LC with small amount of double distilled water was heated up to $65{ }^{\circ} \mathrm{C}$. In order to fill the material into the cell, temperature of the oven was stabilized at $87^{\circ} \mathrm{C}$, i.e., $4-5{ }^{\circ} \mathrm{C}$ below the nematic to isotropic phase transition where the self-diffusion of water into LC is maximum. ${ }^{25}$ The system was then cooled at a very slow rate $\left(0.25^{\circ} \mathrm{C} / \mathrm{min}\right)$ just after the filling, so that homogeneous alignment was obtained, and then at fast rate $\left(5^{\circ} \mathrm{C} / \mathrm{min}\right)$, so that water could not evaporate from the cell. Finally, the cell was sealed from all sides. The tilt angle and textural observations have been carried out by using polarizing microscope (Axioscope-40, Carl Zeiss, Germany).

The Fourier transform infrared (FT-IR) spectra (Nicolet 5700) of CS1026, CS1026/water, and water in the wave number range $500-4000 \mathrm{~cm}^{-1}$ are shown in Figs. 1(a)-1(c), respectively. All the observed bands in the respective spectra can be attributed to the functional groups of their respective systems. It must be stressed that the broad band due to the OH stretching (3000-3800 $\left.\mathrm{cm}^{-1}\right)$ is a good probe of water 


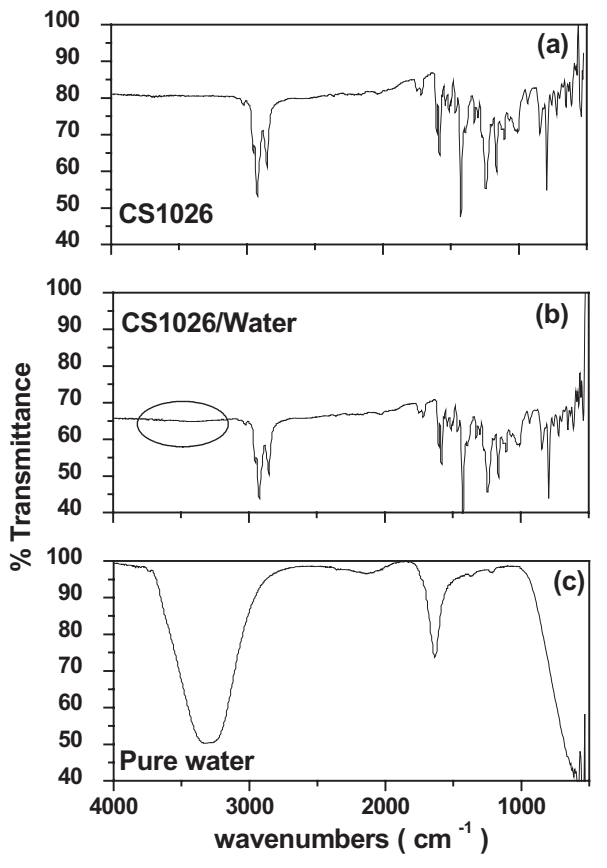

FIG. 1. The FT-IR spectra of (a) CS1026, (b) CS1026/water, and (c) pure water. The encircled area highlights the band (in the range $3000-4000 \mathrm{~cm}^{-1}$ ) due to $\mathrm{OH}$ stretching owing to water in the heterogeneous system.

[Fig. 1(c)]. From an inspection of Fig. 1, it can be noted that the bands representing the CS1026/water system, are negligibly affected by water addition in the range $500-3000 \mathrm{~cm}^{-1}$. The most significant band due to the $\mathrm{OH}$ stretching mode of water $\left(3000-3800 \mathrm{~cm}^{-1}\right)$ in the $\mathrm{LC} /$ water system appears as a very weak band of very low transmittance because the water content in the studied heterogeneous system is very less.

At a temperature $4-5{ }^{\circ} \mathrm{C}$ below the nematic-isotropic phase transition of LC, water may diffuse into LC easily where the diffusion coefficient is higher in comparison to other temperature range. This has been observed in particular for lyotropic LCs. ${ }^{19}$ In our case, it is assumed that not only the higher diffusion coefficient of water but also the reduction in surface tension leads to the diffusion of water between the smectic layers of the FLC. ${ }^{25}$

The most important observation in these LC/water systems is the spatial variation of tilt angle observed in the nonconducting region of the sample where molecules were also observed to switch. This occurrence is here onwards termed as the spatially varying switching phenomenon. This is in contrast to the conventional observation of optical tilt, where change occurs only in between optically transparent electrodes of the LC cells. The switching of molecules under different bias voltages in the nonconducting region of the studied cell is shown in Fig. 2. Both, the nonconducting region (central) and the conducting section are marked in the figure. This circular ringlike pattern was so chosen to demonstrate the in-plane isotropic nature of tilt gradient. The scattering state, which is the state under no bias, is apparent [Fig. 2(a)]. As the field is increased gradually, one can observe the gradient in the optical contrast of the nonconducting region when going away from the conducting region. A completely switched bright state was achieved on application of $30 \mathrm{~V}$ bias [Fig. 2(d)], whereas in case of pure FLC this phenomenon is not observable up to a measurable distance in

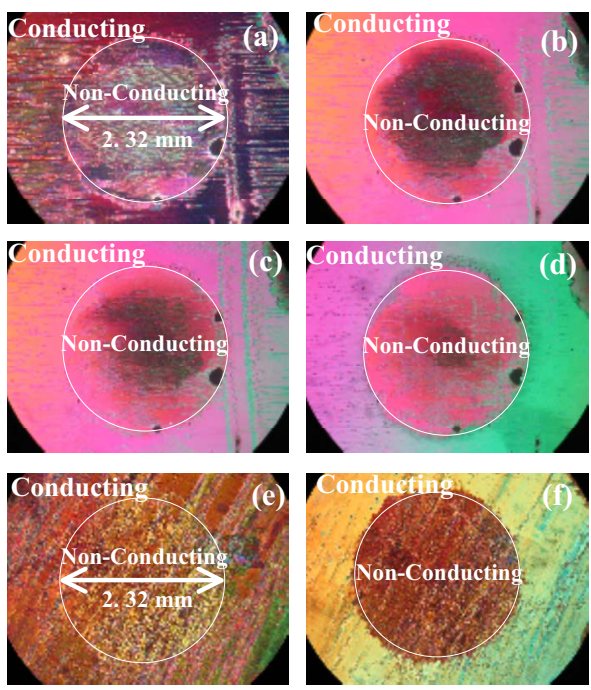

FIG. 2. (Color online) Optical micrographs of FLC/water cell under crossed polarizers at room temperature at (a) $0 \mathrm{~V}$, (b) $4 \mathrm{~V}$, (c) $10 \mathrm{~V}$, (d) $30 \mathrm{~V}$ and micrograph of pure FLC at (e) $0 \mathrm{~V}$ and (f) $30 \mathrm{~V}$ bias.

the nonconducting region [Figs. 2(e) and 2(f)]. Thus, it is clear that there is a gradient in the optical tilt with bias in the nonconducting region of the CS1026/water system only.

To further corroborate this observation, the tilt angle measurement $(2 \theta)$ as a function of distance (from the conduction region) and bias was carried out and shown in Fig. 3. Figure 3(a) shows the variation of tilt angle with distance in the nonconducting region (measured from the conducting edge) at a fixed bias of $10 \mathrm{~V}$ in the circular pattern [refer Fig.
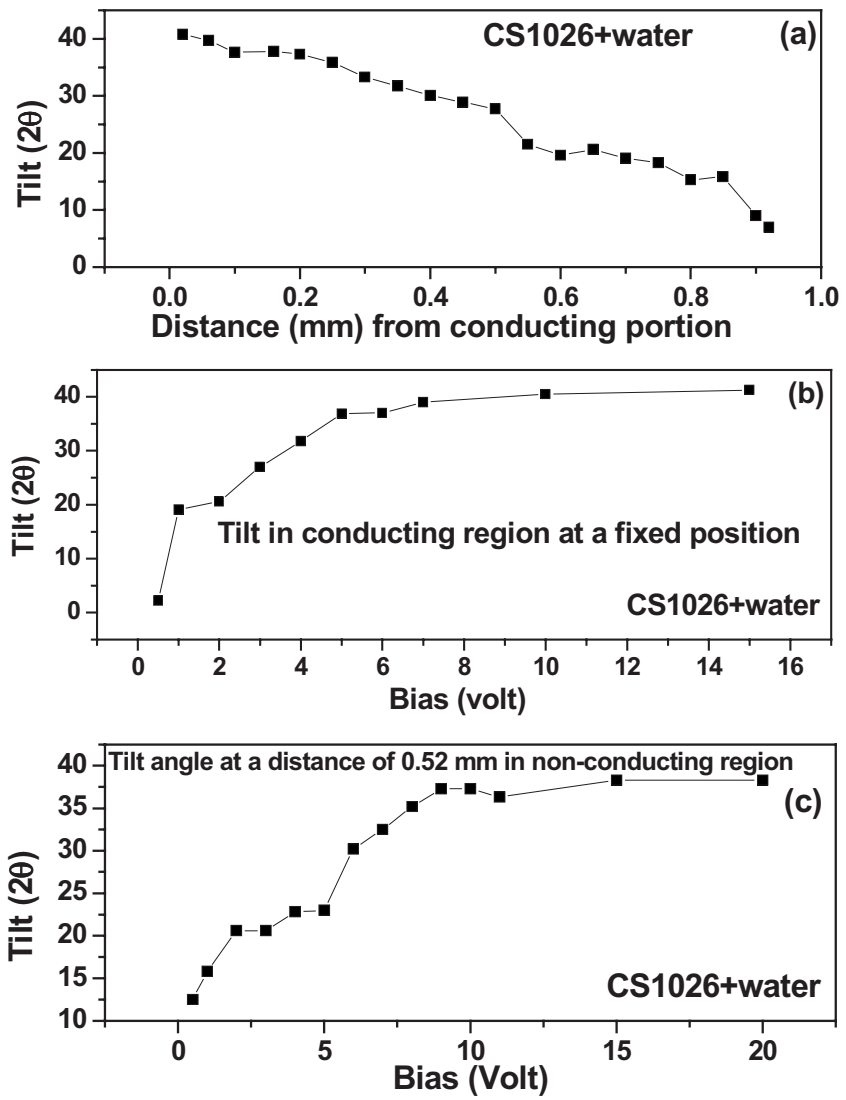

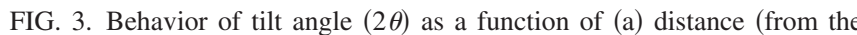
conduction region), (b) bias at a fixed distance in conducting region, and (c) bias at a fixed distance in non-conducting region. 


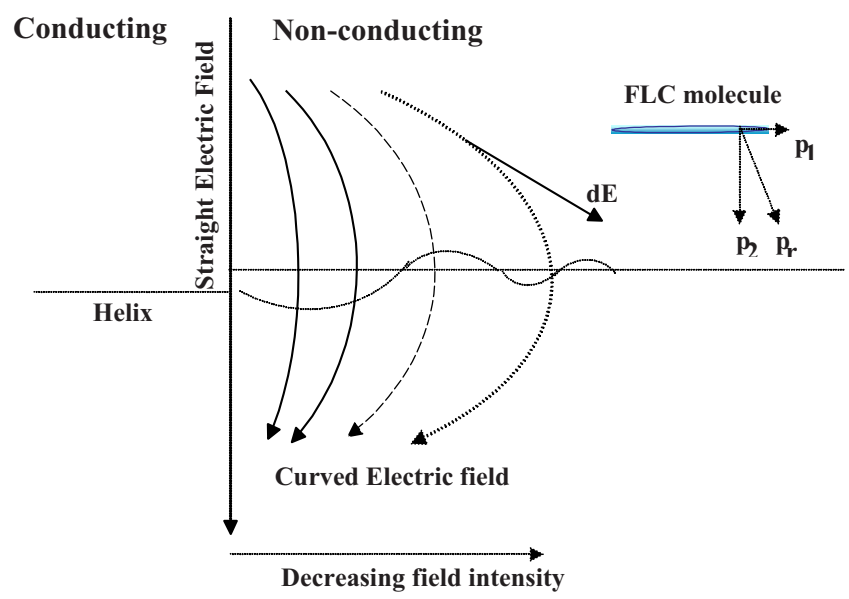

FIG. 4. (Color online) Curving of electric field lines along the edges of the electrodes. The representative molecule showing both the long molecular axes $\left(p_{1}\right)$, short molecular axis $\left(p_{2}\right)$ components of dipole moment, and resultant $\left(p_{r}\right)$ of $p_{1}$ and $p_{2}$.

2(c)]. It shows that the tilt angle is almost equivalent up to a distance close to conducting region and then decreases gradually for distances far off from the conducting edge. The voltage dependent behavior of tilt angle in conducting region [Fig. 3(b)] and at a fixed distance from conducting edge in the nonconducting [Fig. 3(c)] region of the studied cell is also shown. It shows that the saturation of tilt is achieved at a higher dc voltage in case of the nonconducting region. Moreover, it is noted that the amplitude of tilt angle at voltages higher than the saturation voltage is relatively higher in the conducting region. It is evident from the observations that there does exist a field, which interacts with the molecules in nonconducting region but is of a weaker strength. But in case of pure CS1026, since no gradient in the optical contrast of the nonconducting region was observed for a measurable distance [Figs. 2(e) and 2(f)] and not compared with the CS1026/water system.

It is well established that as one moves from conducting region to outside that region, electric field cannot drop from a finite value to a zero value instantaneously so there must be some electric field outside the conducting region, as illustrated in Fig. 4. The electric field lines are curved along the edges of the electrodes. The curvature of these field lines increases with increasing distance from the conducting edge and density of lines increases with field strength. The two dipolar components (along long and short molecular axes) of the molecules seems to have significant role.

From this prototype, it can be inferred that the homogeneously aligned FLC molecules in the conducting region will experience the straight electric field lines while those in the nonconducting region will feel the weak curved electric field lines. The straight electric field lines are coupled with the resultant dipole moment, $p_{r}$ having greater contribution from $p_{2}$ and switch the molecules along the cone as observed in any conventional FLC systems. Whereas in the nonconducting region, the coupling of curved electric field $(d E)$ with $p_{r}$ (with greater role of $p_{1}$ ) leads to the weak switching that possesses spatial variation and so called as spatially varying switching. Though these conditions would exist in case of conventional FLCs as well but due to the presence of water, there is a possibility of diffusion of it in between smectic layers of FLCs leading to the Langmuir monolayer type phenomenon. $^{26,27}$ The formation of Langmuir monolayer provides an interfacial interaction between the two layers of dissimilar surface tensions causing weak long molecular coupling and hence easiness in switching. This reduced surface tension between smectic layers of the system enabling the weak $d E$ field to switch the molecules outside the conducting region, which paves way for another application of FLCs. This phenomenon has also been checked in other FLCs and using different dopants, such as water and ethanol under specific conditions as described in this case.

The authors sincerely thank Dr. Vikram Kumar, Director of the National Physical Laboratory, for continuous encouragement and interest in this work. We sincerely thank Dr. P. Goel, Mr. A. Kumar, and Ms. A. Malik for useful discussions. The authors (A.C., I.C., and J.P.) are thankful to CSIR, New Delhi for financial assistance.

${ }^{1}$ V. Luzzati, H. Mustacchi, and A. Skoulios, Nature (London) 180, 600 (1957).

${ }^{2}$ J. Yamamoto and H. Tanaka, Nature (London) 409, 321 (2001).

${ }^{3}$ N. Nagatani, K. Fukuda, and T. Suzuki, J. Colloid Interface Sci. 234, 337 (2001)

${ }^{4}$ P. Calandra, E. Caponetti, D. C. Martino, P. D’Angelo, A. Minore, and V. T. Liveri, J. Mol. Struct. 522, 165 (2000).

${ }^{5}$ W. Stoeckenius, J. Cell Biol. 12, 221 (1962).

${ }^{6}$ G. Förster, A. Meister, and A. Blume, Curr. Opin. Colloid Interface Sci. 6, 294 (2001).

${ }^{7}$ A. Zheliaskova, R. Marinov, and A. Derzhanski, J. Mol. Struct. 513, 9 (1999).

${ }^{8}$ V. A. Parsegian, Trans. Faraday Soc. 62, 848 (1966).

${ }^{9}$ M. Daoud and C. E. Williams, Soft Matter Physics (Springer, Berlin, 1999).

${ }^{10}$ P. G. de Gennes and J. Prost, Physics of Liquid Crystals, 2nd ed. (Clarendon, Oxford, 1993).

${ }^{11}$ P. Poulin, H. Stark, T. C. Lubensky, and D. A. Weitz, Science 275, 1770 (1997).

${ }^{12}$ V. G. Nazarenko, A. B. Nych, and B. I. Lev, Phys. Rev. Lett. 87, 075504 (2001).

${ }^{13}$ A. Fernández-Nieves, D. R. Link, D. Rudhardt, and D. A. Weitz, Phys. Rev. Lett. 92, 105503 (2004).

${ }^{14}$ F. P. Nicoletta, D. Cupelli, G. De Filpo, and G. Chidichimo, Appl. Phys. Lett. 84, 4260 (2004).

${ }^{15}$ J. M. Brake, M. K. Daschner, Y.-Y. Luk, and N. L. Abbott, Science 302, 2094 (2003).

${ }^{16}$ J.-W. Kim, H. Kim, M. Lee, and J. J. Magda, Langmuir 20, 8110 (2004).

${ }^{17}$ B. Rapp and H. Gruler, Phys. Rev. A 42, 2215 (1990).

${ }^{18}$ M. Ibn-Elhaj, H. Riegler, H. Möhwald, M. Schwendler, and C. A. Helm, Phys. Rev. E 56, 1844 (1997).

${ }^{19}$ F. D. Blum, A. S. Padmanabhan, and R. Mohebbi, Langmuir 1, 127 (1985).

${ }^{20}$ B. M. Ocko, Phys. Rev. Lett. 64, 2160 (1990).

${ }^{21}$ M. P. Valignat, S. Villette, J. Li, R. Barberi, R. Bartolino, E. DuboisViolette, and A. M. Cazabat, Phys. Rev. Lett. 77, 1994 (1996).

${ }^{22}$ P. K. Rai, M. M. Denn, and C. Maldarelli, Langmuir 19, 7370 (2003).

${ }^{23}$ S. A. Harthi and A. K. George, Cryst. Res. Technol. 32, 519 (1997).

${ }^{24}$ Y. R. Martinez-Raton, E. Velasco, A. M. Samoza, L. Mederos, and T. J. Sluckin, J. Chem. Phys. 108, 2583 (1998).

${ }^{25}$ R. Blinc, K. Easwaran, J. Pirs, M. Volfan, and I. Zupancic, Phys. Rev. Lett. 25, 1327 (1970).

${ }^{26}$ Y. Tabe, T. Yamamoto, I. Nishiyama, K. M. Aoki, M. Yoneya, and H. Yokoyama, J. Phys. Chem. B 106, 12089 (2002).

${ }^{27}$ Y. Tabe and H. Yokoyama, Nature Mater. 2, 806 (2003). 